VOLUME 26 (2019) 224-244

DOI: $10.24330 /$ ieja.587081

\title{
ON THE ASSOCIATED PRIME IDEALS AND THE DEPTH OF POWERS OF SQUAREFREE PRINCIPAL BOREL IDEALS
}

\author{
Jürgen Herzog, Bahareh Lajmiri and Farhad Rahmati \\ Received: 24 March 2019; Accepted: 8 May 2019 \\ Communicated by Abdullah Harmancı
}

\begin{abstract}
We study algebraic properties of powers of squarefree principal Borel ideals $I$, and show that $\operatorname{astab}(I)=\operatorname{dstab}(I)$. Furthermore, the behaviour of the depth function depth $S / I^{k}$ is considered.
\end{abstract}

Mathematics Subject Classification (2010): 13F20

Keywords: Squarefree principal Borel ideal, socle, astab, dstab

\section{Introduction}

Borel ideals appear in characteristic zero as generic initial ideals. By applying the stretching operator of Kalai [17] to a Borel ideal, one obtains a squarefree Borel ideal, also called squarefree strongly stable ideal. It has the same graded Betti numbers as the original ideal, see for example [14, Lemma 11.2.6]. This class of ideals was introduced by Hibi and the first author of this paper in [2]. In the sequel, algebraic properties of squarefree strongly stable ideals and their powers have been studied by many authors, see for example [1], [3], [11], [12] and [18].

Among the squarefree strongly stable ideals, the squarefree principal Borel ideals and their powers are best understood. The squarefree principal ideal with Borel generator $u$ is denoted by $B_{S}(u)$. We use some of the known results from Aslam [3], Francisco, Mermin and Schweig [12] and De Negri [7], to get some additional information about the algebraic and homological properties of powers of $B_{S}(u)$. These known facts are recalled in Section 3. In this section we also show that $B_{S}(u)$ is normally torsion free if and only if it is almost normally torsion free, and that this happens if and only if the Borel generator $u$ of the ideal has a specific form, see Corollary 3.4. In Corollary 3.13 we determine the height and bigheight of $B_{S}(u)$, and characterize those squarefree principal Borel ideals which are Cohen-Macaulay. Corollary 3.12 makes more explicit the set $\operatorname{Ass}^{\infty}\left(B_{S}(u)\right)$, as it is described by Aslam in Theorem 3.10 .

For our proofs we essentially use monomial localization. Some of the basic facts about monomial localization are recalled in Section 2. We close this section by 
characterizing in Corollary $2.5 k$-strongly stable ideals. This type of ideals play an important role in Section 4, where the socle of the powers of $B_{S}(u)$ is determined. It turns out, see Corollary 4.2 , that the socle of $B_{S}(u)^{k}$ is a $(k-1)$-stable set of monomials. By using this fact we exhibit in Corollary 4.5 for each $k$ a monomial $w_{k}$ with the property that $\operatorname{depth} S / B_{S}(u)^{k}=0$, if and only if $w_{k} x_{n} \in B_{S}(u)^{k}$. Here $u$ is a squarefree monomial in $S=K\left[x_{1}, \ldots, x_{n}\right]$ with $x_{1} \nmid u$ and $x_{n} \mid u$. These conditions on $u$ are not restrictive, because one can always reduce to this case, see Lemma 5.1 and Proposition 5.2.

The invariant $\operatorname{astab}(u)$ is the smallest number $k$ for which $\operatorname{Ass}\left(B_{S}(u)^{k}\right.$ stabilizes, and $\operatorname{dstab}\left(B_{S}(u)\right)$ is the smallest number $k$ for which depth $S / B_{S}(u)^{k}$ stabilizes. As the main result of Section 5 we show in Theorem 5.5 that for any $u, \operatorname{astab}\left(B_{S}(u)\right)=$ $\operatorname{dstab}\left(B_{S}(u)\right)$. For general monomial ideals $I$, the the Ass-stability and the depth stability are usually unrelated.

For the proof of Theorem 5.5 we use the interesting fact, shown in Theorem 5.4, that the depth stability of monomial localizations of $B_{S}(u)$ are bounded by the depth stability of $B_{S}(u)$.

In the last section we study the depth of $S / B_{S}(u)^{k}$ as a function of $k$. For short we set $f(k)=\operatorname{depth} S / B_{S}(u)^{k}$. Since all powers of $B_{S}(u)^{k}$ have a linear resolution it follows from [13, Proposition 2.1] that $f(k) \leq f(k-1)$ for $k \geq 1$. We also know that the depth function $f(k)$ becomes constant for $k \geq \operatorname{deg} u$, see Proposition5.3. By considering many example it seems to be the case that $f(k)<f(k-1)$, before $f(k)$ becomes constant. This is true if $\operatorname{deg} u \leq 3$, and follows from Proposition 6.5, where we compute $f(k)$ explicitly for $\operatorname{deg} u \leq 3$. In Proposition 6.2 we show that $f(1)=\operatorname{deg} u-1$, and in Proposition 6.3 we determine all squarefree monomials $u$ for which $f(2)=0$. Finally in Theorem 6.6 we show that $f(2)<f(1)$, as expected, unless $f(1)=f(k)$ for some $k \geq 2$, and in this case $f(k)$ is a constant function.

\section{Preliminaries}

In this section we introduce some concepts and results which are important in this paper.

Monomial localizations. In this paper, monomial localizations are an important tool. Let $K$ be a field and let $S=K\left[x_{1}, \ldots, x_{n}\right]$ be the polynomial ring in $n$ variables. Let $T \subset[n]$. We define the monomial prime ideal $P_{T}$ to be the ideal $\left(x_{j}: j \in T^{c}\right)$. Here $T^{c}=[n] \backslash T$ and $[n]=\{1, \ldots, n\}$. We also set $[0]=\emptyset$. Notice that $P_{[0]}=\left(x_{1}, \ldots, x_{n}\right)$ and $P_{[n]}=(0)$. 
Definition 2.1. Let $I \subset K\left[x_{1}, \ldots, x_{n}\right]$ be a monomial ideal, and let $T \subset[n]$. The monomial localization of $I$ with respect to $P_{T}$ is the monomial ideal $I\left(P_{T}\right) \subset S\left(P_{T}\right)$, where $S\left(P_{T}\right)=K\left[x_{j}: j \in T^{c}\right]$ and $I\left(P_{T}\right)=\varphi(I) S\left(P_{T}\right)$, where $\varphi: S \rightarrow S\left(P_{T}\right)$ is the $K$-algebra homomorphism with $x_{j} \mapsto x_{j}$ if $j \in T^{c}$, and $x_{j} \mapsto 1$ if $j \in T$.

Monomial localization with respect to $P_{T}$ and the usual localization with respect to the prime ideal $P_{T}$ are related as follows:

$$
I S_{P_{T}}=I\left(P_{T}\right) S_{P_{T}} .
$$

This identity justifies the name "monomial localization".

For example, if $I=\left(x_{1} x_{3}, x_{1} x_{4}, x_{2} x_{3} x_{4}, x_{3} x_{5} x_{7} x_{8}\right) \subset S=K\left[x_{1}, \ldots, x_{8}\right]$ and $T=\{3,5\}$, then $S\left(P_{T}\right)=K\left[x_{1}, x_{2}, x_{4}, x_{6}, x_{7}, x_{8}\right]$ and $I\left(P_{T}\right)=\left(x_{1}, x_{2} x_{4}, x_{7} x_{8}\right)$.

If $I=P_{T}$, then $I\left(P_{T}\right)$ is the graded maximal ideal of $S\left(P_{T}\right)$ which we denote by $\mathfrak{m}_{S\left(P_{T}\right)}$.

To simplify notation, we set $I_{(j)}=I\left(P_{\{j\}}\right)$ and $S_{(j)}$ for $S\left(P_{\{j\}}\right)$. Note that $S_{(j)}=K\left[x_{i}: i \in[n] \backslash\{j\}\right]$.

Strongly stable ideals. Let $u=x_{1}^{a_{1}} \cdots x_{n}^{a_{n}}$ be a monomial in $S=K\left[x_{1}, \ldots, x_{n}\right]$. We set $\nu_{i}(u)=a_{i}$ for $i=1, \ldots, n$. Now let $I \subset S=K\left[x_{1}, \ldots, x_{n}\right]$ be a monomial ideal. We denote by $G(I)$ the unique set of monomial generators of $I$. For a given integer $k \geq 1$, we let $I \leq k$ be the ideal generated by all $u \in G(I)$ with $\nu_{i}(u) \leq k$ for $i=1, \ldots, n$.

A monomial $u \in S$ be can be written as $u=x_{i_{1}} x_{i_{2}} \cdots x_{i_{d}}$ with $i_{1} \leq i_{2} \leq \ldots \leq i_{d}$. The monomial $u$ is called squarefree if $i_{1}<i_{2}<\ldots<i_{d}$. A monomial ideal $I$ is called a squarefree monomial ideal if all monomials in $G(I)$ are squarefree. Note that for any monomial ideal, the ideal $I \leq 1$ is a squarefree monomial ideal. Moreover, the ideal $I$ is squarefree if and only if $I=I \leq 1$.

Definition 2.2. Let $I \subset S=K\left[x_{1}, \ldots, x_{n}\right]$ be a monomial ideal, and let $k \geq 1$ be an integer, or $k=\infty$. Then $I$ is called $k$-strongly stable, if

(i) $I=I^{\leq k}$;

(ii) for all $u \in G(I)$ and all integers $1 \leq i<j \leq n$ with $\nu_{j}(u)>0$ and $\nu_{i}(u)<k$ it follows that $x_{i}\left(u / x_{j}\right) \in I$.

The following special cases are of particular interest: let $I$ be a monomial ideal. ( $\alpha$ ) If $k=\infty$, then there is no bound on the exponents and $\infty$-strongly stable is simply called strongly stable. In other words, $I$ is strongly stable, if for $u \in G(I)$ and all $j$ such that $x_{j}$ divides $u$, it follows that $x_{i}\left(u / x_{j}\right) \in I$ for all $i \leq j$. 
( $\beta) I$ is called squarefree strongly stable, if it is 1-strongly stable.

Let $u_{1}, \ldots, u_{m}$ be monomials in $S$ with $\nu_{i}\left(u_{j}\right) \leq k$ for $i=1, \ldots, n$ and $j=$ $1, \ldots, m$. There exists a unique smallest $k$-strongly stable ideal containing $u_{1}, \ldots, u_{m}$ which we denote by $B_{S}^{k}\left(u_{1}, \ldots, u_{m}\right)$. The monomials $u_{1}, \ldots u_{m}$ are called Borel generators of $B_{S}^{k}\left(u_{1}, \ldots, u_{m}\right)$. A monomial ideal $I$ is called $k$-principal Borel if $I=B_{S}^{k}(u)$ for some monomial $u$ with $\nu_{i}(u) \leq k$ for $i=1, \ldots, n$. We call 1 principal Borel ideals also squarefree principal Borel. The $k$-principal Borel ideals appear as powers of squarefree principal Borel ideals, see Theorem 4.1.

To simplify notation, we write $B_{S}\left(u_{1}, \ldots, u_{m}\right)$ for $B_{S}^{1}\left(u_{1}, \ldots, u_{m}\right)$ when $u_{1}, \ldots, u_{m}$ are squarefree monomials. The unique strongly stable ideal containing the monomials $u_{1}, \ldots, u_{m}$ will be denoted by $B_{S}^{\infty}\left(u_{1}, \ldots, u_{m}\right)$.

For example, let $I=B_{S}\left(x_{2} x_{4}, x_{1} x_{3}\right)$. Then $G(I)=\left\{x_{1} x_{2}, x_{1} x_{3}, x_{1} x_{4}, x_{2} x_{3}, x_{2} x_{4}\right\}$.

Let $u, v$ be monomials of same degree, and assume that $\nu_{i}(u) \leq k$ for $i=1, \ldots, n$. Then we write $v \preceq_{k} u$ if $v \in B_{S}^{k}(u)$, and $v \prec_{k} u$ if $v \in B_{S}^{k}(u)$ and $v \neq u$. For $\preceq_{\infty}$ we simply write $\preceq$.

For each $d, \preceq_{k}$ defines a partial order on the set of monomials of degree $d$ whose exponents are bounded by $k$. For example, one has $x_{1}^{2} x_{2}^{3} x_{3} \prec_{3} x_{2}^{3} x_{3}^{3}$. A set $\mathcal{S}$ of monomials of degree $d$ whose exponents are bounded by $k$ is called a $k$-stable set, if for $u \in \mathcal{S}$ and $v \preceq_{k} u$ it follows that $v \in \mathcal{S}$.

Note that $B_{S}^{k}\left(u_{1}, \ldots, u_{m}\right)$ is generated by $\bigcup_{i=1}^{m}\left\{v: v \preceq_{k} u_{i}\right\}$. Therefore,

$$
B_{S}^{k}\left(u_{1}, \ldots, u_{m}\right)=\sum_{i=1}^{m} B_{S}^{k}\left(u_{i}\right) .
$$

Let $v, u \in S$ be monomials of degree $d$, where $v=x_{i_{1}} \cdots x_{i_{d}}$ with $i_{1} \leq i_{2} \leq \cdots \leq$ $i_{d}$ and $u=x_{j_{1}} \cdots x_{j_{d}}$ with $j_{1} \leq j_{2} \leq \cdots \leq j_{d}$. By [14, Lemma 4.2.4] one has

$$
v \preceq u \text { if and only if } i_{k} \leq j_{k} \text { for all } k=1, \ldots, d \text {. }
$$

Remark 2.3. Let $u, v \in S$ be monomials. It is clear that if $v \preceq_{k} u$, then $v \preceq u$. On the other hand, if the exponents of $u$ and $v$ are bounded by $k$ and $v \preceq u$, then $v \preceq_{k} u$. This follows from the next lemma.

Lemma 2.4. Let $u \in S$ be a monomial with $\nu_{i}(u) \leq k$ for $i=1, \ldots, n$. Then

$$
B_{S}^{k}(u)=B_{S}^{\infty}(u)^{\leq k}
$$

Proof. Let $v \in B_{S}^{k}(u)$. Then $v \preceq_{k} u$, and hence $v \preceq u$. Therefore, $v \in B_{S}^{\infty}(u)$. Since the exponent of $v$ is bounded by $k$ it follows that $v \in B_{S}^{\infty}(u)^{\leq k}$. 
Conversely, let $v \in B_{S}^{\infty}(u)^{\leq k}$, and let $v=x_{i_{1}} \cdots x_{i_{d}}$ with $i_{1} \leq i_{2} \leq \cdots \leq i_{d}$ and $u=x_{j_{1}} \cdots x_{j_{d}}$ with $j_{1} \leq j_{2} \leq \cdots \leq j_{d}$.

Let $w$ be a monomial of degree $d$ with $v \preceq w$, and let $w=x_{s_{1}} \cdots x_{s_{d}}$ with $s_{1} \leq s_{2} \leq \cdots \leq s_{d}$. We define

$$
\delta(w, v)=\sum_{l=1}^{d}\left(s_{l}-i_{l}\right) .
$$

Since $v \preceq w$, it follows from (1) that $\delta(w, v) \geq 0$, and we have $\delta(w, v)=0$ if and only if $w=v$.

Let $l+1$ be the smallest integer such that $j_{l+1}>i_{l+1}$. Let $u_{1}=x_{j_{l+1}-1} u / x_{j_{l+1}}$. Then the exponents of $u_{1}$ are bounded by $k$, unless $j_{l+1}-1=j_{l}=j_{l-1}=\cdots=$ $j_{l-k+1}$. Assume $j_{l+1}-1=j_{l}$. By the choice of $l$ it follows that $i_{l-k+1}=\cdots i_{l}$. Since the exponents of $v$ are bounded by $k$, it follows that $i_{l+1}>i_{l}$. Moreover, $j_{l+1}>i_{l+1}$. Then $j_{l+1} \geq i_{l}+2=j_{l}+2$, a contradiction. This shows that indeed the exponents of $u_{1}$ are bounded by $k$, and $v \preceq u_{1}$, and hence $v \in B_{S}^{\infty}\left(u_{1}\right)^{\leq k}$. Since $\delta\left(u_{1}, v\right)<\delta(u, v)$, we may assume by induction that $v \in B_{S}^{k}\left(u_{1}\right) \subset B_{S}^{k}(u)$, because $u_{1} \in B_{S}^{k}(u)$.

An immediate consequence of Lemma 2.4 is

Corollary 2.5. Let $u, v$ be monomials of degree $d$ and assume that $\nu_{i}(u), \nu_{i}(v) \leq k$ for $i=1, \ldots, n$. Let $u=x_{j_{1}} \cdots x_{j_{d}}$ with $j_{1} \leq j_{2} \leq \ldots \leq j_{d}$ and $v=x_{i_{1}} \cdots x_{i_{d}}$ with $i_{1} \leq i_{2} \leq \cdots \leq i_{d}$. Then $v \prec_{k} u$ if and only if $i_{r} \leq j_{r}$ for $r=1, \ldots, d$.

If $L \subset[n], S^{\prime}=K\left[x_{i}: i \in L\right]$ and $u_{1}, \ldots, u_{m} \in S^{\prime}$ are monomials with $\nu_{i}\left(u_{j}\right) \leq k$ for $i \in L$ and $j=1, \ldots, m$. Then $B_{S^{\prime}}^{k}\left(u_{1}, \ldots, u_{m}\right)$ is defined in a similar way as in the case $S^{\prime}=S$. For example, if $n=6$ and $L=\{2,4,6\}$, then $S^{\prime}=K\left[x_{2}, x_{4}, x_{6}\right]$. Let $u=x_{4}^{2} x_{6} \in S^{\prime}$ and $k=2$, then $B_{S^{\prime}}^{2}(u)=\left\{x_{2}^{2} x_{4}, x_{2}^{2} x_{6}, x_{2} x_{4} x_{6}, x_{2} x_{4}^{2}, x_{4}^{2} x_{6}\right\}$.

\section{Associated prime ideals of $B_{S}(u)^{k}$}

In this paper we mainly study squarefree principal Borel ideals. This class of ideals behave well under localization, see [3, Theorem 1.2].

Theorem 3.1. Let $u \in S$ be a squarefree monomial of degree $d, u=x_{i_{1}} x_{i_{2}} \cdots x_{i_{d}}$ with $i_{1}<i_{2}<\ldots<i_{d}$, and let $j$ be an integer with $i_{k-1}<j \leq i_{k}$, where we set $i_{0}=0$. Then $B_{S}(u)_{(j)}=B_{S_{(j)}}(v)$, where $v=u / x_{i_{k}}$.

Corollary 3.2. Let $u \in S$ be a squarefree monomial, $I=B_{S}(u)$ and $P \subset S$ a monomial prime ideal. Then $I(P)$ is a squarefree principal Borel ideal in $S(P)$. 
Proof. Let $P=P_{T}$. Then $I\left(P_{T}\right)$ is the ideal in $S\left(P_{T}\right)$ which is obtained from $I$ by the substitutions $x_{j} \mapsto 1$ for $j \in T$. Thus the result follows by repeated application of Theorem 3.1.

A graded ideal $I \subset S$ is called normally torsionfree, if $\operatorname{Ass}(I)=\operatorname{Ass}\left(I^{k}\right)$ for all $k \geq 1$. Moreover, we call $I$ almost normally torsionfree, if $\operatorname{Ass}\left(I^{k}\right) \subseteq \operatorname{Ass}(I) \cup\{\mathfrak{m}\}$ for all $k$, where $\mathfrak{m}=\left(x_{1}, x_{2}, \ldots, x_{n}\right)$ is the graded maximal ideal of $S$.

Corollary 3.3. Let $u=x_{i} x_{n}$, Then $B_{S}(u)$ is almost normally torsionfree. Moreover, $B_{S}(u)$ is normally torsionfree, if and only if $i=1$.

Corollary 3.4. Let $u=x_{i_{1}} \cdots x_{i_{d-1}} x_{n}$, and let $d \geq 3$. Then the following conditions are equivalent:

(a) $B_{S}(u)$ is normally torsionfree.

(b) $B_{S}(u)$ is almost normally torsionfree.

(c) $u=x_{1} x_{2} \cdots x_{d-1} x_{n}$.

In order to prove Corollary 3.3 and Corollary 3.4, we need the following lemmata.

Lemma 3.5. Let $I$ be normally torsion free and let $w$ be a monomial such that $\operatorname{Supp}(w) \cap \operatorname{Supp}(u)=\emptyset$ for all $u \in G(I)$. Then $w I$ is normally torsion free.

Proof. The assumption imply that

$$
\operatorname{Ass}\left((w I)^{k}\right)=\operatorname{Ass}\left(I^{k}\right) \cup \operatorname{Ass}\left((w)^{k}\right)=\operatorname{Ass}\left(I^{k}\right) \cup \operatorname{Ass}((w)) .
$$

It follows that $\operatorname{Ass}\left((w I)^{k}\right)=\operatorname{Ass}(w I)$ for all $k \geq 1$ if and only if $\operatorname{Ass}\left(I^{k}\right)=\operatorname{Ass}(I)$ for all $k \geq 1$.

Lemma 3.6. Let I be a monomial ideal. Then I is almost normally torsionfree if and only if $I_{(j)}$ is normally torsionfree for all $j$.

Proof. The proof follows from the fact that, similarly to ordinary localizations, one has

$$
P \in \operatorname{Ass}_{S_{(j)}}\left(\left(I_{(j)}\right)^{k}\right) \text { if and only if } P S \in \operatorname{Ass}_{S}\left(I^{k}\right) \text { and } P \subset P_{\{j\}},
$$

see [16, Lemma 1.3].

Proof of Corollary 3.3. Note $B_{S}(u)_{(j)}=B_{S_{(j)}}(v)$, where $v$ is a monomial of degree 1. Therefore, $B_{S_{(j)}}(v)$ is normally torsionfree. By Lemma 3.6, it follows that $B_{S}(u)$ is almost normally torsionfree.

If $i_{1}=1$. Then Lemma 3.5 implies that $\operatorname{Ass}\left(B_{S}(u)^{k}\right)=\left(x_{1}\right) \cup\left(x_{2}, \ldots, x_{n}\right)=$ $\operatorname{Min}(I)$. Therefore, $B_{S}(u)$ is normaly torsionfree. 
Proof of Corollary 3.4. (a) $\Rightarrow$ (b) is trivial. (b) $\Rightarrow$ (c) We prove the assertion by induction on $d$. Let $d=3$ and $u=x_{i_{1}} x_{i_{2}} x_{n}$ with $i_{1}<i_{2}<n$. The assumption implies that $B_{S}(u)_{(1)}=B_{S_{(1)}}(v)$ is normally torsionfree, see Lemma 3.6. Here $v=x_{i_{2} x_{n}}$. Now Corollary 3.3 implies that $i_{2}=2$, and therefore and $u=x_{1} x_{2} x_{n}$.

Now let $d>3$, and let $u=x_{i_{1}} x_{i_{2}} \cdots x_{i_{d-1} x_{n}}$ with $i_{1}<i_{2}<\ldots<i_{d-1}<n$. Then Theorem 3.1 implies that $B_{S}(u)_{(1)}=B_{S_{(1)}}(v)$, where $v=x_{i_{2}} \cdots x_{i_{d-1}} x_{n}$. By Lemma 3.6 it follows that $B_{S_{(1)}}(v)$ is normally torsionfree. Hence by induction hypothesis, we have $v=x_{2} x_{3} \cdots x_{d-1} x_{n}$. Therefore, $u=x_{i_{1}} x_{2} \cdots x_{d-1} x_{i_{d}}$. This implies that $i_{1}=1$, and proves (c).

(c) $\Rightarrow$ (a) Since $u=x_{1} x_{2} \cdots x_{d-1} x_{n}$, it follows that $B_{S}(u)=w\left(x_{d}, \ldots, x_{n}\right)$, where $w=x_{1} x_{2} \cdots x_{d-1}$. Therefore, Lemma 3.5 implies that $B_{S}(u)$ is normally torsionfree.

Let $u \in S$ be a squarefree monomial. Then $B_{S}(u)$ does not have any embedded prime ideals, because $B_{S}(u)$ is a squarefree monomial ideal, and hence a radical ideal. In other words, $\operatorname{Ass}\left(B_{S}(u)\right)=\operatorname{Min}\left(B_{S}(u)\right)$. Here, for any ideal $I \subset S, \operatorname{Min}(I)$ denotes the set of minimal prime ideals of $I$.

Let $B_{S}(u)^{\vee}$ denote the Alexander dual of $B_{S}(u)$. Then $v=x_{i_{1}} \cdots x_{i_{k}} \in$ $G\left(B_{S}(u)^{\vee}\right)$, if and only if $\left(x_{i_{1}}, \ldots, x_{i_{k}}\right) \in \operatorname{Min}\left(B_{S}(u)\right)$, see for example [14, Theorem 1.4.6]. Therefore, the set $\operatorname{Min}_{S}\left(B_{S}(u)\right)$ is determined, once we know the set $G\left(B_{S}(u)^{\vee}\right)$. In [12, Theorem 3.18], $G\left(B_{S}(u)^{\vee}\right)$ has been computed.

Theorem 3.7 (Francisco, Mermin, Schweig). Let $u \in S, u=x_{i_{1}} \cdots x_{i_{d}}$ be $a$ monomial with $i_{1}<i_{2}<\ldots<i_{d}$. Then $B_{S}(u)^{\vee}$ is a squarefree strongly stable ideal with Borel generators $x_{r} \cdots x_{i_{r}}$ for $r=1, \ldots, d$.

This theorem has been generalized to $t$-spread principal Borel ideals, see [1, Theorem 1.2].

Let $I \subset S$ be any ideal. Recall that

$$
\operatorname{height}(I)=\min \{\operatorname{height}(P): P \in \operatorname{Ass}(I)\} \text {, }
$$

and

$$
\operatorname{bigheight}(I)=\max \{\operatorname{height}(P): P \in \operatorname{Ass}(I)\} .
$$

As an immediate consequence of Theorem 3.7 we obtain

Corollary 3.8. Let $u \in S, u=x_{i_{1}} \cdots x_{i_{d}}$ be a monomial with $i_{1}<i_{2}<\ldots<i_{d}$ and let $I=B_{S}(u)$. Then

(a) height $(I)=i_{1}$ and $\operatorname{bigheight}(I)=i_{d}-d+1$.

(b) The following conditions are equivalent: 
(i) height $(I)=\operatorname{bigheight}(I)$.

(ii) $i_{j}=i_{1}+j-1$ for $j=1, \ldots, d$.

(iii) $S / I$ is Cohen-Macaulay.

Proof. (i) $\Longleftrightarrow$ (ii) We have

$i_{1} \leq i_{2}-1 \leq \ldots \leq i_{j}-j+1 \leq i_{j+1}-(j+1)+1 \leq \ldots \leq i_{d}-d+1$.

From (a) it follows that $i_{1}=i_{d}-d+1$. Therefore, $i_{j}-j+1=i_{j+1}-(j+1)+1$ for all $j$ which implies that $i_{j+1}=i_{j}$ for all $j$. This yields the desired conclusion.

(iii) $\Rightarrow$ (i) Since $S / I$ is Cohen-Macaulay, the ideal $I$ is unmixed, see for example [6, Theorem 2.1.6]. Since $I$ is unmixed if and only if height $(I)=\operatorname{bigheight}(I)$, the assertion follows.

(ii) $\Rightarrow$ (iii) By Theorem 3.7, $I^{\vee}=B_{S}\left(x_{d} \cdots x_{i_{d}}\right)$ for ideals satisfying condition (ii). It is known that $B_{S}\left(x_{d} \cdots x_{i_{d}}\right)$ has a linear resolution, see [1, Proposition 2.4]. Therefore, $S / I$ is Cohen-Macaulay, see for example [14, Theorem 8.1.9].

The following results [3, Theorem 2.1 and Corollary 2.3] is also important for this paper. We denote by $\mathfrak{m}$ the graded maximal ideal of $S=K\left[x_{1}, \ldots, x_{n}\right]$. For a monomial $v \neq 1$ we set $\min (v)=\min \{j: j \in \operatorname{supp}(v)\}$ and $\max (v)=\max \{j: j \in$ $\operatorname{supp}(v)\}$. If $v=1$, we set $\min (v)=\max (v)=0$.

Theorem 3.9 (Aslam). Let $u \in S=K\left[x_{1}, \ldots, x_{n}\right]$ be a squarefree monomial of degree $d$ and let $I=B_{S}(u)$. Then $\mathfrak{m} \in \operatorname{Ass}\left(I^{k}\right)$ for some $k$ if and only if $\min (u)>1$ and $\max (u)=n$. If this is the case, then $\mathfrak{m} \in \operatorname{Ass}\left(I^{k}\right)$ for all $k \geq d$.

An alternate proof of this fact is given in Proposition 4.6.

By Brodmann ([4] and [5]), for any graded ideal $I \subset S=K\left[x_{1}, \ldots, x_{n}\right]$, there exists an integer $k_{0}$ such that $\operatorname{Ass}\left(I^{k}\right)=\operatorname{Ass}\left(I^{k_{0}}\right)$ for all $k \geq k_{0}$. We set $\operatorname{Ass}^{\infty}(I)=$ $\operatorname{Ass}\left(I^{k_{0}}\right)$.

In our case, $I=B_{S}(u)$ with $u=x_{i_{1}} \cdots x_{i_{d}}$ and $i_{1}<i_{2}<\ldots<i_{d}$. Therefore, $I$ is a squarefree monomial ideal, and hence $\operatorname{Ass}(I)=\operatorname{Min}(I)$. Since $\operatorname{Min}(I) \subset \operatorname{Ass}^{\infty}(I)$, it follows in our case that $\operatorname{Ass}(I) \subset \operatorname{Ass}^{\infty}(I)$. In Corollary 3.4, we have seen that $\operatorname{Ass}(I)=\operatorname{Ass}^{\infty}(I)$ if and only if $u=x_{1} x_{2} \cdots x_{d-1} x_{i_{d}}$.

Let $T \subset[n]$. By using Theorem 3.1 and induction on $|T|$ it follows that there exists a (unique) monomial $u_{T} \subset S_{T}$ of degree $d-|T|$ such that $B_{S}(u)_{T}=B_{S_{T}}\left(u_{T}\right)$.

For example, if $u=x_{2} x_{5} x_{6} x_{7} x_{9}$ and $T=\{3,5,9\}$, then

$$
u_{T}=u_{359}=\left(u_{9}\right)_{35}=\left(\left(x_{2} x_{5} x_{6} x_{7}\right)_{5}\right)_{3}=\left(x_{2} x_{6} x_{7}\right)_{3}=x_{2} x_{7} .
$$

Note that the monomial prime ideal $P$ belongs to $\operatorname{Ass}^{\infty}(I)$ if and only if $\mathfrak{m}_{P} \in$ $\operatorname{Ass}_{S(P)}\left(I(P)^{k}\right)$, for all $k \gg 0$. Here $\mathfrak{m}_{P}=P S(P)$ denotes the graded maximal 
ideal of $S(P)$. By Corollary 3.2 the monomial localization $I(P)$ of the squarefree principal monomial $I$ is again a squarefree principal monomial ideal.

A graded ideal $I \subset S$ is said to satisfy the persistence property, if $\operatorname{Ass}_{S}\left(I^{k}\right) \subset$ $\operatorname{Ass}_{S}\left(I^{k+1}\right)$ for all $k \geq 1$. Since squarefree principal monomial ideals satisfy the persistence property (see [1, Corollary 2.6]), we conclude that $P$ belongs to $\operatorname{Ass}^{\infty}(I)$ if and only if $\mathfrak{m}_{P} \in \operatorname{Ass}_{S(P)}\left(I(P)^{k}\right)$ for some $k$. From this observation together with Theorem 3.9 one obtains [3, Theorem 3.2].

Theorem 3.10 (Aslam). Let $T$ be any subset of $[n]$ and assume that $\min (u)>$ 1. Then $P_{T} \in \operatorname{Ass}^{\infty}\left(B_{S}(u)\right)$ if and only if $\min \left(T^{c}\right)<\min \left(u_{T}\right)$ and $\max \left(u_{T}\right)=$ $\max \left(T^{c}\right)$.

For the next result we need

Lemma 3.11. Suppose $\min (u)>1$. Then $\min \left(T^{c}\right)<\min \left(u_{T}\right)$ if and only if $u_{T} \neq 1$.

Proof. It is enough to show that $\min \left(T^{c}\right)<\min \left(u_{T}\right)$ if $u_{T} \neq 1$. The other direction is trivial. We use induction on $|T|$. If $|T|=0$, the assertion is trivial, since $\min (u)>1$ and $T^{c}=[n]$. Now let $|T|>0$. Note that $T \neq[n]$, because we assume that $u_{T} \neq 1$. This means that $T$ is a non-empty proper subset of $[n]$. Let $u=x_{i_{1}} x_{i_{2}} \cdots x_{i_{d}}$ with $i_{1}<i_{2}<\ldots<i_{d}$ and let $T=\left\{j_{1}, j_{2}, \ldots, j_{s}\right\}$ with $j_{1}<j_{2}<\ldots<j_{s}$. Let $r$ be the biggest number such that $j_{i}=i$ for $i=1, \ldots, r-1$. Then $\min \left(T^{c}\right)=r$, and

$$
u_{T}=\left(x_{i_{r}} \cdots x_{i_{d}}\right)_{\left\{j_{r}, \ldots, j_{s}\right\}}
$$

by Theorem 3.1. Therefore, $\min \left(u_{T}\right) \geq i_{r} \geq r+1$. Thus we see that $\min \left(T^{c}\right)=$ $r<r+1 \leq \min \left(u_{T}\right)$, as desired.

Corollary 3.12. Let $u=x_{i_{1}} \cdots x_{i_{d}}$ be a monomial in $S=K\left[x_{1}, \ldots, x_{n}\right]$ with $i_{1}<i_{2}<\ldots<i_{d}$, and $T=\left\{j_{1}, \ldots, j_{s}\right\}$ with $j_{1}<j_{2}<\ldots<j_{s}$. Suppose that $i_{j}=j$ for $j=1, \ldots, r$ and $i_{r+1}>r+1$. Then

$$
\operatorname{Ass}^{\infty}\left(B_{S}(u)\right)=\left\{\left(x_{1}\right), \ldots,\left(x_{r}\right)\right\} \cup\left\{P_{T}:[r] \subseteq T, u_{T} \neq 1, \max \left(T^{c}\right)=\max \left(u_{T}\right)\right\} .
$$

Moreover, $u_{T} \neq 1$ if and only if $s<d$ or $s \geq d$ and $j_{l}>i_{l}$ for some $l$.

Proof. By assumption we have $u=x_{1} \cdots x_{r} u^{\prime}$, where $u^{\prime}=x_{i_{r+1}} \cdots x_{i_{d}}$ with $i_{r+1}>$ $r+1$. Therefore, $B_{S}(u)=x_{1} \cdots x_{r} B_{S^{\prime}}\left(u^{\prime}\right) S$, where $S^{\prime}=K\left[x_{r+1}, \ldots, x_{n}\right]$. Hence (2) implies that $\operatorname{Ass}^{\infty}\left(B_{S}(u)\right)=\left\{\left(x_{1}\right), \ldots,\left(x_{r}\right)\right\} \cup \operatorname{Ass}^{\infty}\left(B_{S^{\prime}}\left(u^{\prime}\right)\right) S$. Since $\min \left(u^{\prime}\right)>$ $r+1$, we may apply Theorem 3.10 to $u^{\prime}$ in $S^{\prime}$. Therefore, the result follows from Theorem 3.10 and Lemma 3.11. 
Corollary 3.13. Let $u \in S, u=x_{i_{1}} \cdots x_{i_{d}}$ be a monomial with $i_{1}<i_{2}<\ldots<i_{d}$ and let $I=B_{S}(u)$. Suppose that $i_{j}=j$ for $j=1, \ldots, r$ and $i_{r+1}>r+1$. Then bigheight $\left(I^{l}\right)=i_{d}-r$ for $l \gg 0$.

Proof. Since $l \gg 0$, it follows that bigheight $\left(I^{l}\right)=\max \left\{\right.$ height $\left.P_{T}: P_{T} \in \operatorname{Ass}^{\infty}(I)\right\}$. The ideal $P_{T}$ for $T=[r]$ has height $i_{d}-r$, and this the largest possible height among the ideals $P_{T}$. Since $u_{T}=x_{i_{r+1}} \cdots x_{i_{d}}$, Corollary 3.12 implies that $P_{T} \in \operatorname{Ass}^{\infty}(I)$.

Example 3.14. Let $u=x_{1} x_{3} x_{5}$ and $I=B_{S}(u)$. Then

$$
\left\{T:[1] \subseteq T, u_{T} \neq 1, \max \left(T^{c}\right)=\max \left(u_{T}\right)\right\}=\{\{1\},\{1,2\},\{1,3\},\{1,4,5\}\} .
$$

Hence

$$
\operatorname{Ass}^{\infty}(I)=\left\{\left(x_{1}\right)\right\} \cup\left\{\left(x_{2}, x_{3}, x_{4}, x_{5}\right),\left(x_{3}, x_{4}, x_{5}\right),\left(x_{2}, x_{4}, x_{5}\right),\left(x_{2}, x_{3}\right)\right\} .
$$

\section{The socle of $S / B_{S}(u)^{k}$}

In this section we determine the powers of $B_{S}(u)$ and their socle. We first recall a result of De Negri [7, Proposition 3.4], and present a proof it by using sortability. This makes the proof substantially shorter.

Theorem 4.1. Let $u \in S$ be a squarefree monomial. Then $B_{S}(u)^{k}=B_{S}^{k}\left(u^{k}\right)$ for all $k \geq 1$. In particular, $B_{S}(u)^{k}$ is k-strongly stable.

Proof. Let $u=x_{l_{1}} \cdots x_{l_{d}}$ with $l_{1} \leq l_{2} \leq \cdots \leq l_{d}$, and suppose that $v \in B_{S}^{k}\left(u^{k}\right)$, and let $v=x_{i_{1}} \cdots x_{i_{k d}}$ with $i_{1} \leq i_{2} \leq \cdots \leq i_{k d}$. Since $v \preceq_{k} u^{k}$ it follows that $i_{(r-1) k+1}, \ldots, i_{r k} \leq l_{r}$ for $r=1, \ldots, d$, see Corollary 2.5.

For $j=1, \ldots, k$, let $v_{j}=\prod_{r=1}^{d} x_{i(r-1) k+j}$. Then $v=v_{1} \cdots v_{k}$ and $v_{j} \preceq u$. It remains that to be shown that each $v_{j}$ is squarefree. Suppose $v_{j}$ is not squarefree. Then there exists $r$ with $1 \leq r<d$ such that $x_{i_{(r-1) k+j}}=x_{i_{r k+j}}$. Hence $i_{(r-1) k+j}=$ $i_{(r-1) k+j+1}=\cdots=i_{r k+j}$. This implies that $\nu_{i(r-1) k+j}(v)>k$, a contradiction.

Conversely, let $v \in B_{S}(u)^{k}$. By [1, Proposition 2.4], $G\left(B_{S}(u)\right)$ is sortable. Therefore, $v=v_{1} v_{2} \cdots v_{k}$ with $v_{j} \in B_{S}(u)$ and $\left(v_{i}, v_{j}\right)$ is sorted for all $i, j$ with $1 \leq i<$ $j \leq k$. It follows from $[9,(6.3)]$, that if $v_{1}=x_{i_{1}} \cdots x_{i_{d}}$ with $i_{1}<i_{2}<\ldots<i_{d}$, $v_{2}=x_{j_{1}} \cdots x_{j_{d}}$ with $j_{1}<j_{2}<\ldots<j_{d}, \ldots, v_{k}=x_{s_{1}} \cdots x_{s_{d}}$ with $s_{1}<s_{2}<\ldots<$ $s_{d}$, then $i_{1} \leq j_{1} \leq \ldots \leq s_{1} \leq i_{2} \leq j_{2} \leq \ldots \leq s_{2} \leq \ldots \leq i_{k} \leq j_{k} \leq \ldots \leq s_{k}$. From this it follows that $v \in B_{S}^{\infty}\left(u^{k}\right)$. Since each $v_{j}$ is squarefree it follows that $v \in B_{S}^{k}\left(u^{k}\right)$. 
For any monomial ideal we denote by $\operatorname{Soc}(S / I)$ the (finite) set of monomial $v \in S$ with $v \notin I$ and $v x_{j} \in I$ for $j=1, \ldots, n$. Note that depth $S / I=0$ if and only if $\operatorname{Soc}(S / I) \neq \emptyset$.

Corollary 4.2. Let $u \in S$ be a squarefree monomial of degree $d$, and let $I=B_{S}(u)$. Suppose that depth $S / I^{k}=0$. Then $\operatorname{Soc}\left(S / I^{k}\right)$ is a $(k-1)$-stable set of monomials generated in degree $k d-1$.

Proof. By [1, Proposition 2.4] the ideal $I^{k}$ has $k d$-linear resolution. Suppose now that depth $S / I^{k}=0$. Then the graded minimal free resolution of $S / I^{k}$ is of the form

$$
0 \rightarrow F_{n} \rightarrow F_{n-1} \rightarrow \cdots \rightarrow F_{1} \rightarrow F_{0} \rightarrow S / I^{k} \rightarrow 0
$$

with $F_{0}=S$ and $F_{i}=S(-k d-i+1)^{\beta_{i}}$ for $i=1, \ldots, n$. So $F_{n}=S(-k d-n+1)^{\beta_{n}}$. This shows that all elements of $\operatorname{Soc}\left(S / I^{k}\right)$ have degree $(k d+n-1)-n=k d-1$. Indeed, $F_{n} / \mathfrak{m} F_{n}$ and the Koszul homology $H_{n}\left(x_{1}, \ldots, x_{n} ; S / I^{k}\right)$ are isomorphic as the graded $K$-vector spaces. The generators of $H_{n}\left(x_{1}, \ldots, x_{n} ; S / I^{k}\right)$ are $v e_{1} \wedge \ldots \wedge e_{n}$ with $v \in \operatorname{Soc}\left(S / I^{k}\right)$. This gives us the above formula for the degree of the socle elements.

By [15, Corollary 1.2], $\nu_{i}(v) \leq k-1$ for all $v \in \operatorname{Soc}\left(S / I^{k}\right)$ and $i=1, \ldots, n$. Thus it remains to be shown that if $v \in \operatorname{Soc}\left(S / I^{k}\right), x_{j} \mid v, i<j$ and $\nu_{i}\left(x_{i} v\right) \leq k-1$, then $v_{0}:=x_{i}\left(v / x_{j}\right) \in \operatorname{Soc}\left(S / I^{k}\right)$, that is, $x_{l} v_{0} \in I^{k}$ for $l=1, \ldots, n$. Indeed, if $l=j$, then $x_{l} v_{0}=x_{i} v \in I^{k}$. If $l \neq j$, then $x_{l} v_{0}=x_{i}\left(x_{l} v\right) / x_{j} \in I^{k}$ because $x_{l} v \in I^{k}$ and $I^{k}$ is $k$-stable, see Theorem 4.1 .

Let $1<d<n$ be integers and for $k \geq 2$ let $c_{k}, r_{k}$ be integers such that

$$
k d-1=c_{k}(k-1)+r_{k} \quad \text { with } \quad 0 \leq r_{k}<k-1 .
$$

Then we define the monomial $w_{k}=x_{1}^{k-1} \cdots x_{c_{k}}^{k-1} x_{c_{k}+1}^{r}$. Note $w_{k} \in S$ if and only if $c_{k} \leq n$ when $r=0$, and $c_{k}+1 \leq n$ if $r \neq 0$.

Corollary 4.3. Let $u \in S$ be a squarefree monomial of degree $d$, and let $I=B_{S}(u)$. Then depth $S / I^{k}=0$ if and only if $w_{k} x_{n} \preceq u^{k}$.

Proof. If depth $S / I^{k}=0$, then $\operatorname{Soc}\left(S / I^{k}\right)$ is a non-empty $(k-1)$-stable set. Let $v \in \operatorname{Soc}\left(S / I^{k}\right)$. Then $\left\{w: w \preceq_{k-1} v\right\} \subseteq \operatorname{Soc}\left(S / I^{k}\right)$. Since $w_{k} \preceq_{k-1} v$ it follows that $w_{k} \in \operatorname{Soc}\left(S / I^{k}\right)$. Therefore, $w_{k} x_{n} \in I^{k}=B_{S}^{k}\left(u^{k}\right)$. This implies that $w_{k} x_{n} \preceq_{k} u^{k}$, and hence $w_{k} x_{n} \preceq u^{k}$, see Remark 2.3.

Conversely, suppose that $w_{k} x_{n} \preceq u^{k}$. Therefore, $x_{i} w_{k}=x_{i}\left(w_{k} x_{n}\right) / x_{n} \preceq u^{k}$. Since the exponents of $w_{k}$ are bounded by $k-1$, the exponents of $x_{i} w_{k}$ are bounded 
by $k$. Therefore, by Remark $2.3, x_{i} w_{k} \preceq_{k} u^{k}$ for all $i$. This means that $x_{i} w_{k} \in I^{k}$ for all $i$. Hence $w_{k} \in \operatorname{Soc}\left(S / I^{k}\right)$, and so depth $S / I^{k}=0$.

Note that $w_{k} x_{n} \preceq u^{k}$ only if $w_{k} \in S$.

Remark 4.4. Let $d<n, f_{d}(k)=\lceil(k d-1) /(k-1)\rceil$ and $w_{k}=x_{1}^{k-1} \cdots x_{c_{k}}^{k-1} x_{c_{k}+1}^{r}$. Then

(a) $f_{d}(k)=c_{k}$ if $r=0$ and $f_{d}(k)=c_{k}+1$ if $r \neq 0$. Therefore, $w_{k} \in S$ if and only if $f_{d}(k) \leq n$.

(b) The function $f_{d}(k)$ is a non-increasing function with $f_{d}(k) \leq n$ for $k \geq d$.

(c) Let $k_{0}=\min \left\{k: f_{d}(k) \leq n\right\}$. Then $k_{0} \leq d$.

Proof. (a) follows from the definition of $c_{k}$, see (4).

(b) It is obvious that $f_{d}(k)$ is a non-increasing function. Since $f_{d}(d)=d+1 \leq n$, we also have $f_{d}(k) \leq n$ for $k \geq d$.

(c) follows from (b).

Corollary 4.5. Let $k_{0}$ be defined as in Remark 4.5. Then $\min \left\{k: \operatorname{depth} S / I^{k}=\right.$ $0\} \geq k_{0}$. In particular, this lower bound for $\min \left\{k: \operatorname{depth} S / I^{k}=0\right\}$ depends only on $d$ and $n$.

In general the inequality $\min \left\{k: \operatorname{depth} S / I^{k}=0\right\} \geq k_{0}$ may be strict, as the following example shows: Let $u=x_{2} x_{3} x_{4} x_{5} x_{6} \in S=K\left[x_{1}, \ldots, x_{6}\right]$. Then $d=5$ and $n=6$. Then $f_{5}(2)=9, f_{5}(3)=7$ and $f_{5}(4)=6$. Therefore, $k_{0}=4$, but on the other hand 5 is the smallest number $k$ for which depth $S / I^{k}=0$.

We use this characterization of the socle elements to show

Proposition 4.6. Let $I=B_{S}\left(x_{i_{1}} x_{i_{2}} \cdots x_{i_{d}}\right) \subset S=K\left[x_{1}, \ldots, x_{n}\right]$ with $1<i_{1}<$ $i_{2}<\ldots<i_{d}=n$. Then $\mathfrak{m} \in \operatorname{Ass}\left(I^{k}\right)$ for $k \geq d$.

Proof. It is enough to show that $\mathfrak{m} \in \operatorname{Ass}\left(I^{d}\right)$, since $I$ satisfies the persistence property, see [1, Proposition 2.4].

Note that $w_{d}=x_{1}^{d-1} \cdots x_{d+1}^{d-1}$. We show that $w_{d} x_{n} \in I^{d}$. From Corollary 4.5 it then follows that $\operatorname{depth}\left(S / I^{d}\right)=0$ which implies that $\operatorname{dstab}(I) \leq d$. To see that $w_{d} x_{n} \in I^{d}$, we must show that $w_{d} x_{n} \prec_{d} u^{d}$, where $u=x_{i_{1}} \cdots x_{i_{d}}$ with $1<i_{1}<i_{2}<\ldots i_{d}=n$. Since $v=x_{2} \cdots x_{d} x_{n} \prec_{1} u$ it follows $v^{d} \prec_{d} u^{d}$. Therefore, it suffices to show that $w_{d} x_{n} \prec_{d} v^{d}$.

We write $w_{d} x_{n}=x_{k_{1}} \ldots x_{k_{d^{2}}}$ with $k_{1} \leq k_{2} \leq \ldots \leq k_{d^{2}}$ and $v^{d}=x_{l_{1}} \cdots x_{l_{d^{2}}}$ with $l_{1} \leq l_{2} \leq \ldots \leq l_{d^{2}}$. Since $\nu_{r}\left(w_{d} x_{n}\right), \nu_{r}\left(v^{d}\right) \leq d$ for all $r$, we must show that $k_{r} \leq l_{r}$ 
for $r=1, \ldots, d^{2}$, see Corollary 2.5. Let $r$ be an integer with $1 \leq r \leq d^{2}$. We may assume that $r \leq(d-1) d$, because $l_{r}=n$, for $(d-1) d<r \leq d^{2}$. Let

$r-1=q d+t_{1}$ with $0 \leq t_{1} \leq d-1$, and $r-1=q^{\prime}(d-1)+t_{2}$ with $0 \leq t_{2} \leq d-2$.

Then $l_{r}=q+2$ and $k_{r}=q^{\prime}+1$. Now $k_{r} \leq l_{r}$ if and only if $q^{\prime}-q \leq 1$. Indeed, we have $q d+t_{1}=q^{\prime}(d-1)+t_{2}$. This implies that $d\left(q^{\prime}-q\right)=t_{1}-t_{2}+q^{\prime}<2 d$. Therefore, $q^{\prime}-q<2$, as desired.

\section{Comparison of $\operatorname{astab}\left(B_{S}(u)\right)$ with $\operatorname{dstab}\left(B_{S}(u)\right)$}

As mentioned in Section 3, for any graded ideal $I \subset S=K\left[x_{1}, \ldots, x_{n}\right]$ there exists an integer $k_{1}$ such that $\operatorname{Ass}_{S}\left(I^{k}\right)=\operatorname{Ass}_{S}\left(I^{k_{1}}\right)$ for all $k \geq k_{1}$. The smallest integer $k_{1}$ with this property is denoted by $\operatorname{astab}(I)$. Similarly there exists an integer $k_{2}$ such that depth $S / I^{k}=\operatorname{depth} S / I^{k_{2}}$ for all $k \geq k_{2}$. The smallest integer $k_{2}$ with this property is denoted by $\operatorname{dstab}(I)$. The purpose of the section is to compute $\operatorname{dstab}(I)$ and $\operatorname{astab}(I)$ when $I$ is a squarefree principal Borel ideal.

Let $I=B_{S}\left(x_{i_{1}} x_{i_{2}} \cdots x_{i_{d}}\right) \subset S=K\left[x_{1}, \ldots, x_{n}\right]$ with $i_{1}<i_{2}<\ldots<i_{d}$. Assume that $i_{d}=m \leq n$, and let $S^{\prime}=K\left[x_{1}, \ldots, x_{m}\right]$ and $J=B_{S^{\prime}}\left(x_{i_{1}} x_{i_{2}} \cdots x_{i_{d}}\right)$. Then, we obviously one gets

Lemma 5.1. With the assumptions and notation introduced, we have

$$
\operatorname{depth} S / I^{k}=\operatorname{depth} S^{\prime} / J^{k}+n-m \text { for all } k,
$$

and

$$
\operatorname{Ass}_{S}\left(I^{k}\right)=\left\{P S: P \in \operatorname{Ass}_{S^{\prime}}\left(J^{k}\right)\right\} \quad \text { for all } k .
$$

In particular, $\operatorname{dstab}(I)=\operatorname{dstab}(J)$ and $\operatorname{astab}(I)=\operatorname{astab}(J)$.

Therefore, for the rest of this section, if not otherwise stated, we may assume that $i_{d}=n$.

Next we show

Lemma 5.2. Let $I=B_{S}(u) \subset S=K\left[x_{1}, \ldots, x_{n}\right]$, where $u=x_{i_{1}} x_{i_{2}} \cdots x_{i_{d}}$ with $1=i_{1}<i_{2}<\ldots<i_{d}=n$. Let $J=B_{S^{\prime}}\left(u^{\prime}\right) \subset S^{\prime}=K\left[x_{2}, \ldots, x_{n-1}\right]$, where $u^{\prime}=u / x_{1}$. Then for all $k$,

$$
\operatorname{Ass}_{S}\left(I^{k}\right)=\left\{P S: P \in \operatorname{Ass}_{S^{\prime}}\left(J^{k}\right)\right\} \cup\left(x_{1}\right) \quad \text { and } \quad \operatorname{depth} S / I^{k}=\operatorname{depth} S^{\prime} / J^{k}+1 .
$$

In particular, $\operatorname{dstab}(I)=\operatorname{dstab}(J)$ and $\operatorname{astab}(I)=\operatorname{astab}(J)$. 
Proof. We observe that $I^{k}=x_{1}^{k} \tilde{J}^{k}$ for all $k$, where $\tilde{J}=J S$. Therefore, the statement about $\operatorname{Ass}_{S}\left(I^{k}\right)$ follows from (2). Since $\left(x_{1}^{k}\right) \cap \tilde{J}^{k}=x_{1}^{k} \tilde{J}^{k}$ we get the short exact sequence

$$
0 \rightarrow S / I^{k} \rightarrow S /\left(x_{1}^{k}\right) \oplus S / \tilde{J}^{k} \rightarrow S /\left(x_{1}^{k}, \tilde{J}^{k}\right) \rightarrow 0 .
$$

By the depth lemma (see [8, Corollary 18.6]) we have

$$
\begin{aligned}
\operatorname{depth} S / I^{k} & \geq \min \left\{\operatorname{depth}\left(S /\left(x_{1}^{k}\right) \oplus S / \tilde{J}^{k}\right), \operatorname{depth} S /\left(x_{1}^{k}, \tilde{J}^{k}\right)+1\right\} \\
& =\min \left\{\operatorname{depth} S /\left(x_{1}^{k}\right), \operatorname{depth} S / \tilde{J}^{k}, \operatorname{depth} S /\left(x_{1}^{k}, \tilde{J}^{k}\right)+1\right\} .
\end{aligned}
$$

Since $x_{1}^{k}$ is regular on $S / \tilde{J}^{k}$, it follows that $\operatorname{depth} S /\left(x_{1}^{k}, \tilde{J}^{k}\right)=\operatorname{depth} S / \tilde{J}^{k}-1$. Therefore, $\operatorname{depth} S / I^{k} \geq \operatorname{depth} S / \tilde{J}^{k}$.

By using again the depth lemma we also get $\operatorname{depth} S / \tilde{J}^{k}-1=\operatorname{depth} S /\left(x_{1}^{k}, \tilde{J}^{k}\right) \geq \min \left\{\operatorname{depth} S /\left(x_{1}^{k}\right), \operatorname{depth} S / \tilde{J}^{k}, \operatorname{depth} S / I^{k}-1\right\}$. This implies that $\operatorname{depth} S / \tilde{J}^{k} \geq \operatorname{depth} S / I^{k}$, and hence $\operatorname{depth} S / I^{k}=\operatorname{depth} S / \tilde{J}^{k}$. Since $\tilde{J}=J S$, it follows that $\operatorname{depth} S / \tilde{J}=\operatorname{depth} S^{\prime} / J$. This yields the desired conclusion.

Because of Lemma 5.2 we may also assume for the rest of the section that $i_{1}>1$.

Combining Lemma 5.2 with Corollary 4.5 we obtain

Proposition 5.3. Let $I=B_{S}(u) \subset S=K\left[x_{1}, \ldots, x_{n}\right]$ with $u=x_{i_{1}} x_{i_{2}} \cdots x_{i_{d}}$ and $1<i_{1}<i_{2}<\ldots<i_{d}=n$. Then we have:

(a) $\operatorname{dstab}(I)=\min \left\{k: \operatorname{depth} S / I^{k}=0\right\}$.

(b) Let $k_{0}$ be defined as in Corollary 4.5. Then

$$
\operatorname{dstab}(I)=\min \left\{k \geq k_{0}: w_{k} x_{n} \in I^{k}\right\} .
$$

(c) $k_{0} \leq \operatorname{dstab}(I) \leq \operatorname{astab}(I) \leq d$.

The following result tells us how dstab behaves under monomial localization.

Theorem 5.4. Let $I=B_{S}(u) \subset S=K\left[x_{1}, \ldots, x_{n}\right]$ with $u=x_{i_{1}} x_{i_{2}} \cdots x_{i_{d}}$ and $1<i_{1}<i_{2}<\ldots<i_{d}=n$. Then

$$
\operatorname{dstab}\left(I_{(a)}\right) \leq \operatorname{dstab}(I) \quad \text { for } \quad a=1, \ldots, n \text {. }
$$

Proof. We first consider the case that $a \leq i_{d-1}$. Then $I_{(a)}=B_{S}(u)_{(a)}=B_{\left.S_{(} a\right)}\left(u_{a}\right)$ and $\min \left(u_{a}\right)>\min \left(T^{c}\right)$ and $\max \left(u_{a}\right)=\max \left(T^{c}\right)=n$. Then Proposition 4.6 implies that depth $S / I^{k}=0$ for some $k$ and $\operatorname{depth} S_{(a)} / I_{(a)}^{l}=0$ for some $l$. Let $k$ and $l$ be the smallest numbers with this property. Then $k=\operatorname{dstab}(I)$ and $l=\operatorname{dstab}\left(I_{(a)}\right)$, and we want to show that $l \leq k$. For this it is enough to show that $\operatorname{depth} S_{(a)} / I_{(a)}^{k}=$ 
0 . Since depth $S / I^{k}=0$, Corollary 4.5 implies that $w_{k} x_{n} \in I^{k}$. By Theorem 4.1, $I^{k}=B_{S}^{k}\left(u_{k}\right)$. Therefore, $w_{k} x_{n} \preceq_{k} u^{k}$. Let $w_{k} x_{n}=x_{t_{1}(k)} x_{t_{2}(k)} \ldots x_{t_{k d}(k)}$ with $t_{1}(k) \leq \ldots \leq t_{k d}(k)$ and $u^{k}=x_{l_{1}(k)} x_{l_{2}(k)} \cdots x_{l_{k d}(k)}$ with $l_{1}(k) \leq \cdots \leq l_{k d}(k)$. By Corollary $2.5, w_{k} x_{n} \preceq u^{k}$ if and only if $t_{s}(k) \leq l_{s}(k)$ for $s=1, \ldots, k d$. The numbers $l_{s}(k)$ and $t_{s}(k)$ can be computed.

With the notation introduced we have

$$
l_{s}(k)=i_{j}, \quad \text { where } \quad j=\lceil s / k\rceil,
$$

and

$$
t_{s}(k)= \begin{cases}\lceil s /(k-1)\rceil, & \text { if } s \leq(k-1) c \\ c+1, & \text { if }(k-1) c<s<k d \\ n, & \text { if } s=k d\end{cases}
$$

Let $b$ be the unique number with $i_{b-1}<a \leq i_{b}$. Then $u_{a}=u / i_{b} \in S_{(a)}$ is of degree $d-1$, see Theorem 3.1. Let $w_{k}^{\prime} \in S_{(a)}$ be the socle test element for $I_{(a)}^{k}$ in $S_{(a)}$. We have depth $S_{(a)} / I_{(a)}^{k}=0$, once we have shown that $w_{k}^{\prime} x_{m} \preceq_{k} u_{a}^{k}$.

Let

$$
w_{k}^{\prime} x_{n}=x_{t_{1}^{\prime}(k)} x_{\left.t_{2}^{\prime} k\right)} \ldots x_{t_{k(d-1)}^{\prime}(k)} \quad \text { with } \quad t_{1}^{\prime}(k) \leq \ldots \leq t_{k(d-1)}^{\prime}(k),
$$

and

$$
u_{a}^{k}=x_{l_{1}^{\prime}(k)} x_{l_{2}^{\prime}(k)} \cdots x_{l_{k(d-1)}^{\prime}(k)} \quad \text { with } \quad l_{1}^{\prime}(k) \leq \cdots \leq l_{k(d-1)}^{\prime}(k) .
$$

It remains to be shown that $t_{s}^{\prime}(k) \leq l_{s}^{\prime}(k)$ for $s=1, \ldots, k(d-1)$.

Note that

$$
l_{s}^{\prime}(k)= \begin{cases}l_{s}(k), & \text { if } s \leq k(b-1) \\ l_{s+k}(k), & \text { if } k(b-1)<s \leq k(d-1),\end{cases}
$$

and

$$
t_{s}^{\prime}(k)= \begin{cases}t_{s}(k), & \text { if } s \leq(k-1)(a-1) \\ t_{s+k-1}(k), & \text { if }(k-1)(a-1)<s \leq k(d-1) .\end{cases}
$$

Note that $b \leq a$, because $b-1 \leq i_{b-1}<a$. We consider different cases.

Case 1: $s \leq(k-1)(a-1)$ : Then, since $b \leq a$, we have

$$
\begin{aligned}
t_{s}^{\prime}(k) & =t_{s}(k) \leq l_{s}(k)= \begin{cases}l_{s}^{\prime}(k), & \text { if } s \leq k(b-1) ; \\
l_{s-k}^{\prime}(k), & \text { if } k(b-1)<s \leq k(d-1),\end{cases} \\
& \leq l_{s}^{\prime}(k),
\end{aligned}
$$

because $l_{s}^{\prime}(k)$ is a non-decreasing function.

Case 2: $s>(k-1)(a-1)$ : Then

$$
t_{s}^{\prime}(k)=t_{s+k-1}(k) \leq l_{s+k-1}(k)= \begin{cases}l_{s+k-1}^{\prime}(k), & \text { if } s \leq k(b-1) ; \\ l_{s-1}^{\prime}(k), & \text { if } k(b-1)<s \leq k(d-1),\end{cases}
$$

Therefore, if $s>k(b-1)$, then $t_{s}^{\prime}(k) \leq l_{s}^{\prime}(k)$. 
Suppose now that $(k-1)(a-1)<s<k(b-1)$. Then $t_{s}(b)=\lceil s /(k-1)\rceil \geq a$ and $l_{s}(k)=i_{j}$, where $j=\lceil s / k\rceil$. Therefore, $l_{s}(k)=i_{c}$ with $c \leq b-1$. Hence,

$$
a \leq t_{s}(k) \leq l_{s}(k)=i_{c} \leq i_{b-1},
$$

a contradiction. So this case cannot happen.

It remains to treat the case that $a>i_{d-1}$. Then $u_{a}=x_{i_{1}} \cdots x_{i_{d-1}}$, and $I_{(a)}=$ $B_{S_{(a)}}\left(u_{a}\right)$. Let $J=B_{S^{\prime}}\left(u_{a}\right)$, where $S^{\prime}=k\left[x_{1}, \ldots, x_{i_{d-1}}\right]$. Then, by Lemma 5.1 , $\operatorname{dstab}(J)=\operatorname{dstab}\left(I_{(a)}\right)$, and furthermore by Proposition 5.3 we have $\operatorname{dstab}(J)=$ $\min \left\{k: \operatorname{depth} S^{\prime} / J^{k}=0\right\}$. Therefore, we must show that $\operatorname{depth} S^{\prime} / J^{k}=0$ if $\operatorname{depth} S / I^{k}=0$. Since $\operatorname{depth} S / I^{k}=0$ it follows $w_{k} x_{n} \preceq_{k} u^{k}$. Let $w_{k}=$ $\prod_{s=1}^{k d-1} x_{t_{s}(k)}$. Then the socle test element for $J^{k}$ is $w_{k}^{\prime}=\prod_{s=1}^{k(d-1)-1} x_{t_{s}(k)}$. Then it is clear that $w_{k}^{\prime} x_{i_{d-1}} \preceq_{k} u_{a}^{k}$. This shows that depth $S^{\prime} / J^{k}=0$, as desired.

As the main result of the section we show

Theorem 5.5. Let $S=K\left[x_{1}, \ldots, x_{n}\right]$ be the polynomial ring in $n$ variables, let $u \in S$ be a squarefree monomial ideal and let $I=B_{S}(u)$. Then $\operatorname{astab}(I)=\operatorname{dstab}(I)$.

Proof. Let $u=x_{i_{1}} \cdots x_{i_{d}}$ with $i_{1}<i_{2}<\cdots<i_{d}$. By the discussion at the beginning of this section and by Lemma 5.2 we may assume that $1<i_{1}$ and $i_{d}=n$. Let $k=\operatorname{dstab}(I)$. By Proposition 5.3, we have $\mathfrak{m} \in \operatorname{Ass}_{S}\left(I^{k}\right)$. Now let $P \in \operatorname{Ass}_{S}^{\infty}(I)$ with $P \neq \mathfrak{m}$. Then there exists $j$ and $P^{\prime} \in \operatorname{Ass}_{S_{(j)}}^{\infty}\left(I_{(j)}\right)$ with $P=P^{\prime} S$. By induction we may assume that $\operatorname{astab}\left(I_{(j)}\right)=\operatorname{dstab}\left(I_{(j)}\right)$. Thus, if $k^{\prime}=\operatorname{dstab}\left(I_{(j)}\right)$, then $P^{\prime} \in \operatorname{Ass}_{S_{(j)}}\left(I_{(j)}^{k^{\prime}}\right)$. Therefore, $P \in \operatorname{Ass}_{S}\left(I^{k^{\prime}}\right)$, by (3). Theorem 5.4 implies that $k^{\prime} \leq k$. Since squarefree principal monomial ideals satisfy the persistence property, we conclude that $P \in \operatorname{Ass}_{S}\left(I^{k}\right)$, as desired. This shows that $\operatorname{astab}(I) \leq \operatorname{dstab}(I)$. The other inequality is shown in Proposition 5.3.

\section{On the depth of $S / B_{S}(u)^{k}$}

We provide some partial results regarding depth $S / I^{k}$ for $I=B_{S}(u)$. Since all powers have a linear resolution it follows that $\operatorname{depth} S / I^{k+1} \leq \operatorname{depth} S / I^{k}$ for all $k \geq 1$, as mentioned in the proof of Proposition 5.3. Actually all powers of $I$ have linear quotients for a suitable order, as shown in [1, Proposition 2.4]. Here we show

Proposition 6.1. Let $I=B_{S}(u)$. Then for all $k$, the ideal $I^{k}$ has linear quotients with respect to the lexicographic order induced by $x_{1}>x_{2}>\cdots>x_{n}$.

Proof. Let $v, w \in I^{k}$ with $w>v$ with respect to the lexicographic order. Let $v=x_{j_{1}} x_{j_{2}} \cdots x_{j_{k d}}$ with $j_{1} \leq j_{2} \leq \ldots \leq j_{k d}$, and $w=x_{l_{1}} x_{l_{2}} \cdots x_{l_{k d}}$ with $l_{1} \leq l_{2} \leq$ $\ldots \leq l_{k d}$. Since $w>v$ there exists an integer $r$ such that $l_{s}=j_{s}$ for $s=1, \ldots, r-1$ 
and $j_{r}>l_{r}$. Then $x_{l_{r}}$ divides $w / \operatorname{gcd}(w, v)$. Let $w^{\prime}=x_{l_{r}} v / x_{j_{r}}$. Since $l_{r}<j_{r}$ it follows that $w^{\prime}>v$ and $w^{\prime} / \operatorname{gcd}\left(w^{\prime}, v\right)=x_{l_{r}}$ which divides $w / \operatorname{gcd}(w, v)$. It remains to be shown that $w^{\prime} \in I^{k}$. To see that we use Theorem 4.1 which says $I^{k}=B_{S}^{k}\left(u^{k}\right)$. Since $v \in B_{S}^{k}\left(u^{k}\right)$ it follows that $v \prec_{k} u^{k}$. We now show that $w^{\prime} \prec_{k} v$. Then it follows $w^{\prime} \prec_{k} u^{k}$, and hence belongs to $I^{k}$. Indeed, since $x_{j_{r}}$ in $v$ is replaced by $x_{l_{r}}$ with $l_{r}<j_{r}$ to obtain $w^{\prime}$ it follows that $w^{\prime} \prec v$. Let $w^{\prime}=x_{f_{1}} x_{f_{2}} \cdots x_{f_{k d}}$ with $f_{1} \leq f_{2} \leq \ldots \leq f_{k d}$. Then $f_{s}=j_{s}$ for $s \neq r$ and $f_{r}=l_{r}$. To have $w^{\prime} \prec_{k} v$ we must show that there is no $t$ such that $f_{t}=f_{t+1}=\cdots=f_{t+k}$, or equivalently there is no $t$ such that $f_{t}=f_{t+k}$. If $t+k<r$ or $t>r$ we have $f_{t}=j_{t}$ and $f_{t+k}=j_{t+k}$. Therefore, $f_{t} \neq f_{t+k}$ because the exponents of the monomial $v$ are bounded by $k$. Now let $t \leq r \leq t+k$. Suppose first that $r<t+k$. Then $r+1 \leq t+k$, and $f_{r+1}=j_{r+1} \geq j_{r}>l_{r}=f_{r}$. So not all $f_{s}$ are the same for $s$ with $t \leq s \leq t+k$. Finally, assume that $r=t+k$ and $f_{r-k}=\cdots=f_{r}$. For $s<r$ we have $f_{s}=j_{s}=l_{s}$ and $f_{r}=l_{r}$. Thus our assumption implies that $l_{r-k}=\cdots=l_{r}$, a contradiction because $w \in B_{S}^{k}\left(u^{k}\right)$.

In all examples considered, the depth function $f(k)=\operatorname{depth} S / I^{k}$ is strictly decreasing until it becomes stable. In some special case we show that this is indeed the case.

We first observe

Proposition 6.2. Let $I=B_{S}(u)$ with $u=x_{i_{1}} x_{i_{2}} \cdots x_{i_{d}}$ and $i_{1}<i_{2}<\cdots<i_{d}=n$. Then $\operatorname{depth} S / I=d-1$.

Proof. We have to show that $\operatorname{proj} \operatorname{dim} I=n-d$. Let $\tau$ be the inverse of the spreading operator $\sigma$. By definition, if $v=x_{k_{1}} x_{k_{2}} \cdots x_{k_{d}}$ with $k_{1}<k_{2}<\cdots<$ $k_{d}$, then $\tau(v)=\prod_{j=1}^{d} x_{k_{j}-(j-1)}$, and when $I$ is a squarefree monomial ideal with $G(I)=\left\{v_{1}, \ldots, v_{m}\right\}$, one sets $I^{\tau}$ to be the ideal with $G\left(I^{\tau}\right)=\left\{\tau\left(v_{1}\right), \ldots, \tau\left(v_{m}\right)\right\}$.

By [10, Proposition 2.1] we have $B_{S}(u)^{\tau}=B_{S}^{\infty}(\tau(u))$, where $B_{S}^{\infty}(\tau(u))$ is the principal Borel ideal with Borel generator $\tau(u)$. It follows from [10, Theorem 1.11] that $\operatorname{proj} \operatorname{dim} B_{S}(u)=\operatorname{proj} \operatorname{dim} B_{S}^{\infty}(\tau(u))$. Note that all generators of $B_{S}^{\infty}(\tau(u))$ belong to $T=K\left[x_{1}, \ldots, x_{n-d+1}\right]$. Let $J=G\left(B_{S}^{\infty}(\tau(u))\right) T$. Then $B_{S}^{\infty}(\tau(u))=J S$. It follows that

$$
\operatorname{depth} S / I=\operatorname{depth} S / B_{S}^{\infty}(\tau(u))=\operatorname{depth} T / J+d-1 .
$$

It remains to be shown that $\operatorname{depth} T / J=0$. It is well-known and easy to prove that

$$
J=\prod_{j=1}^{d}\left(x_{1}, \ldots, x_{i_{j}-(j-1)}\right) .
$$


Since $i_{d}=n$ it follows that $J=J_{0} \mathfrak{n}$, where $J_{0}=\prod_{j=1}^{d-1}\left(x_{1}, \ldots, x_{i_{j}-(j-1)}\right)$ and $\mathfrak{n}$ is the maximal ideal of $T$. This shows that any minimal generator of $J_{0}$ defines a socle element of $T / J$. In particular, $\operatorname{depth} T / J=0$.

The next result tells us when depth $S / I^{2}=0$ for $I=B_{S}(u)$.

Proposition 6.3. Let $u=x_{i_{1}} x_{i_{2}} \cdots x_{i_{d}} \subset S$ be a monomial with $1<i_{1}<i_{2}<$ $\ldots<i_{d}=n$, and let $I=B_{S}(u)$. The following conditions are equivalent:
(a) $\operatorname{depth} S / I^{2}=0$.
(b) $x_{1} \cdots x_{2 d-1} \in \operatorname{Soc}\left(S / I^{2}\right)$.
(c) $i_{j} \geq 2 j$ for $j=1, \ldots, d-1$.

Proof. (a) $\Longleftrightarrow$ (b) follows from Corollary 4.3.

(b) $\Longleftrightarrow(\mathrm{c}) w_{2}=x_{1} \cdots x_{2 d-1} \in \operatorname{Soc}\left(S / I^{2}\right)$, if and only if $x_{1} \cdots x_{2 d-1} x_{n} \in I^{2}=$ $B_{S}\left(u^{2}\right)$, and this is the case if and only if

$$
x_{1} \cdots x_{2 d-1} x_{n} \prec u^{2}=x_{i_{1}} x_{i_{1}} x_{i_{2}} x_{i_{2}} \cdots x_{i_{d-1}} x_{i_{d-1}} x_{n} x_{n} .
$$

By Corollary 2.5 this is the case if and only if $i_{j} \geq 2 j$ for $j=1, \ldots, d-1$, as desired.

Corollary 6.4. Let $I=B_{S}\left(x_{i} x_{n}\right)$. Then $\operatorname{depth} S / I=1$, and for $k \geq 2$ we have

$$
\operatorname{depth} S / I^{k}= \begin{cases}1, & \text { if } i=1 \\ 0, & \text { if } i>1\end{cases}
$$

Proof. The fact $\operatorname{depth}(S / I)=1$ follows from Proposition 6.2. In order to compute $\operatorname{depth}\left(S / I^{k}\right)$, we first suppose that $i=1$. Then Lemma 5.2 implies that $\operatorname{depth} S / I^{k}=\operatorname{depth} S^{\prime} / B_{S^{\prime}}\left(x_{n-1}\right)^{k}+1$. Since $B_{S^{\prime}}\left(x_{n-1}\right)=\left(x_{1}, \ldots, x_{n-1}\right)$, it follows that depth $S / I^{k}=1$ for all $k$. Finally, for $i>1$ and $k>1$, the result follows from Proposition 6.3.

Next we consider the case $d=3$.

Proposition 6.5. Let $I=B_{S}\left(x_{i} x_{j} x_{n}\right)$ with $i<j<n$.

(a) If $i=1$, then depth $S / I=2$, and for $k \geq 2$ we have

$$
\operatorname{depth} S / I^{k}= \begin{cases}2, & \text { if } j=2, \\ 1, & \text { if } j>2 .\end{cases}
$$

(b) If $i>1$, then $\operatorname{depth} S / I=2$, and $\operatorname{depth} S / I^{k}=0$ for $k \geq 3$. Moreover,

$$
\operatorname{depth} S / I^{2}= \begin{cases}1, & \text { if } j=3, \\ 0, & \text { if } j>3 .\end{cases}
$$


Proof. (a) follows from Lemma 5.2 and Proposition 6.4.

(b) Proposition 6.2 shows that $\operatorname{depth} S / I=2$. Moreover, $\operatorname{depth} S / I^{k}=0$ for $k \geq 3$, see Proposition 5.3. Thus it remains to consider the case $k=2$. If $j>3$, then depth $S / I^{2}=0$. Finally assume that $j=3$. Then $x_{i} x_{j} x_{n}=x_{2} x_{3} x_{n}$. Then $\operatorname{depth} S / I^{2}>0$, by Proposition 6.3. On the other hand, depth $S / I^{2}<\operatorname{depth} S / I=$ 2 , by the next Theorem 6.6. Therefore, $\operatorname{depth} S / I^{2}=1$, as desired.

The following result supports what we expect, namely that depth $S / I^{k}<\operatorname{depth} S / I^{k-1}$ if $k \leq \operatorname{astab}(I)$.

Theorem 6.6. Let $I=B_{S}(u)$. Then the following conditions are equivalent:

(a) $u=x_{1} x_{2} \cdots x_{d-1} x_{n}$.

(b) $\operatorname{depth} S / I=\operatorname{depth} S / I^{k}$ for all $k$.

(c) $\operatorname{depth} S / I=\operatorname{depth} S / I^{k}$ for some $k \geq 2$.

(d) $\operatorname{depth} S / I=\operatorname{depth} S / I^{2}$.

Moreover, if one of these equivalent conditions fails, then $\operatorname{depth} S / I^{2}<\operatorname{depth} S / I$.

For the proof of Theorem 6.6 we use

Lemma 6.7. Let $I=B_{S}(u)$, where $u=x_{i_{1}} \cdots x_{i_{d}}$ with $1<i_{1}<i_{2}<\ldots<i_{d}=n$. Let $v \in I^{2}$ and let $J=\left(w \in G\left(I^{2}\right): v<_{\operatorname{lex}} w\right)$. Assume that $x_{n}$ divides $v$. Then

$$
\left\{x_{i}: i<n \text { and } x_{i}^{2} \nmid v\right\} \subseteq J: v .
$$

Proof. Let $i$ be such that $i<n$ and $x_{i}^{2} \nmid v$, and let $w=v x_{i} / x_{n}$. Then $\nu_{j}(w) \leq 2$ for all $j$ and $w \prec v$. Therefore, $w \in I^{2}$. Since $v<_{\operatorname{lex}} w$, it follows that $w \in J$. Moreover, $v x_{i}=w x_{n}$, and hence $x_{i} \in J: v$.

Proof of Theorem 6.6. (a) $\Rightarrow$ (b) Note that $I=x_{1} \cdots x_{d-1}\left(x_{d}, \ldots, x_{n}\right)$. Therefore, $I^{k} \cong\left(x_{d}, \ldots, x_{n}\right)^{k}$, and hence depth $S / I^{k}=\operatorname{depth} I^{k}-1=\operatorname{depth}\left(x_{d}, \ldots, x_{n}\right)^{k}-$ $1=\operatorname{depth} S /\left(x_{d}, \ldots, x_{n}\right)^{k}$. Since $\operatorname{depth} K\left[x_{d}, \ldots, x_{n}\right] /\left(x_{d}, \ldots, x_{n}\right)^{k}=0$, it follows that

$$
\operatorname{depth} S / I^{k}=\operatorname{depth} S /\left(x_{d}, \ldots, x_{n}\right)^{k}=d-1=\operatorname{depth} S / I .
$$

(b) $\Rightarrow$ (c) is trivial.

(c) $\Rightarrow$ (d) Since $\operatorname{depth} S / I \geq \operatorname{depth} S / I^{2} \geq \operatorname{depth} S / I^{k}$, it is obvious that (c) implies (d).

(d) $\Rightarrow$ (a) We show that if $u \neq x_{1} \cdots x_{d-1} x_{n}$, then $\operatorname{depth} S / I^{2}<\operatorname{depth} S / I$. Since $u \neq x_{1} \cdots x_{d-1} x_{n}$ it follows that $u=x_{1} x_{2} \cdots x_{i} v$ with $i<d-1$ and $v=$ $x_{j_{1}} x_{j_{2}} \cdots x_{n}$ with $j_{1}<j_{2}<\cdots<n$ and $j_{1}>i+1 \geq 1$. Then $I=B_{S}(u)=$ $x_{1} \cdots x_{i} J$, where $J=B_{S}(v)$. From this it follows that $\operatorname{depth} S / I^{2}<\operatorname{depth} S / I$ if 
and only if depth $S / J^{2}<\operatorname{depth} S / J$. Therefore, we may assume that $u=x_{i_{1}} \cdots x_{i_{d}}$ with $1<i_{1}<i_{2}<\cdots<i_{d}=n$.

We claim that there exits $v \in G\left(I^{2}\right)$ such that $x_{n}$ divides $v$ and such that $|\mathcal{S}| \geq n-d+1$, where $\mathcal{S}=\left\{x_{i}: i<n\right.$ and $\left.x_{i}^{2} \nmid v\right\} \mid$. By Lemma 6.7, the claim implies that $\operatorname{proj} \operatorname{dim} I^{2} \geq n-d+1$, and hence $\operatorname{depth} S / I^{2} \leq d-2<\operatorname{depth} S / I$.

We write $u^{2}=x_{l_{1}} \cdots x_{l_{2 d}}$ with $l_{1} \leq l_{2} \leq \cdots \leq l_{2 d}$, and let $v=x_{t_{1}} x_{t_{2}} \cdots x_{t_{2 d}}$ with $t_{1} \leq t_{2} \leq \ldots \leq t_{2 d}$.

For the proof of the claim we consider two cases.

Case 1: $i_{j}=i_{1}+j-1$ for $j=1, \ldots, d$. Then we let $t_{j}=j$ for $j=1, \ldots, i_{1}$ and $t_{j}=l_{j}$ for $j=i_{1}+1, \ldots, 2 d$. Then $|\mathcal{S}| \geq i_{1}=n-d+1$, because $n=i_{1}+d-1$.

Case 2: There exists $k$ such that $i_{k+1}>i_{k}+1$. Then for all $j$ we let $t_{j}=l_{j}-1$ if $j$ is odd, and let $t_{j}=l_{j}$ if $j$ is even. Then $v$ is divided by as many squares as we have integers $j$ with $i_{j+1}=i_{j}+1$. Therefore, $v$ has at most $d-1$ squares as factors. This implies that $|\mathcal{S}| \geq n-d+1$, as desired.

\section{References}

[1] C. Andrei, V. Ene and B. Lajmiri, Powers of t-spread principal Borel ideals, Arch. Math. (Basel), 112(6) (2019), 587-597.

[2] A. Aramova, J. Herzog and T. Hibi, Squarefree lexsegment ideals, Math. Z., 228(2) (1998), 353-378.

[3] A. Aslam, The stable set of associated prime ideals of a squarefree principal Borel ideal, Bull. Math. Soc. Sci. Math. Roumanie (N.S.), 57(105) (2014), 243252 .

[4] M. Brodmann, Asymptotic stability of $\operatorname{Ass}\left(M / I^{n} M\right)$, Proc. Amer. Math. Soc., 74(1) (1979), 16-18.

[5] M. Brodmann, The asymptotic nature of the analytic spread, Math. Proc. Cambridge Philos. Soc., 86 (1979), 35-39.

[6] W. Bruns and J. Herzog, Cohen-Macaulay Rings, Cambridge Studies in Advanced Mathematics, 39, Cambridge University Press, Cambridge, 1993.

[7] E. De Negri, Toric rings generated by special stable sets of monomials, Math. Nachr., 203(1) (1999), 31-45.

[8] D. Eisenbud, Commutative Algebra: with a view toward algebraic geometry, Graduate Texts in Mathematics, 150, Springer-Verlag, New York, 1995.

[9] V. Ene and J. Herzog, Gröbner Bases in Commutative Algebra, Graduate Studies in Mathematics, 130, American Mathematical Society, Providence, RI, 2012. 
[10] V. Ene, J. Herzog and A. Asloob Qureshi, t-spread strongly stable ideals, arXiv:1805.02368 [math.AC].

[11] C. A. Francisco, Minimal graded Betti numbers and stable ideals, Comm. Algebra, 31(10) (2003), 4971-4987.

[12] C. A. Francisco, J. Mermin and J. Schweig, Borel generators, J. Algebra, 332(1) (2011), 522-542.

[13] J. Herzog and T. Hibi, The depth of powers of an ideal, J. Algebra, 291(2) (2005), 534-550.

[14] J. Herzog and T. Hibi, Monomial Ideals, Graduate Texts in Mathematics, 260, Springer-Verlag London, Ltd., London, 2011.

[15] J. Herzog and T. Hibi, Bounding the socles of powers of squarefree monomial ideals, Commutative Algebra and Noncommutative Algebraic Geometry, Vol. II, Math. Sci. Res. Inst. Publ., 68, Cambridge Univ. Press, New York, (2015), 223-229.

[16] J. Herzog, A. Rauf and M. Vladoiu, The stable set of associated prime ideals of a polymatroidal ideal, J. Algebraic Combin., 37(2) (2013), 289-312.

[17] G. Kalai, Algebraic shifting, in: Computational Commutative Algebra and Combinatorics, (Osaka, 1999), Adv. Stud. Pure Math., 33, Math. Soc. Japan, Tokyo, (2002), 121-163.

[18] I. Peeva and M. Stillman, The minimal free resolution of a Borel ideal, Expo. Math., 26(3) (2008), 237-247.

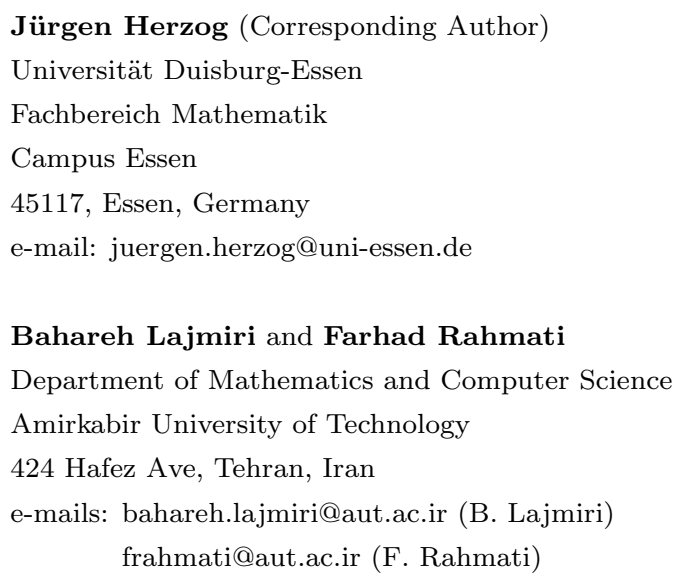

